\title{
Aplicações da espectroscopia no infravermelho no controle de qualidade de medicamentos contendo diclofenaco de potássio. Parte I : Dosagem por regressão multivariada
}

\author{
Jorcen Simon de Souza' ${ }^{1}$, Marco Flôres Ferrão ${ }^{1,2, \text { * }}$ \\ ${ }^{1}$ Grupo de Quimiometria, ${ }^{2}$ Programa de Pós-Graduação em Sistemas e Processos Industriais, \\ Universidade de Santa Cruz do Sul - UNISC
}

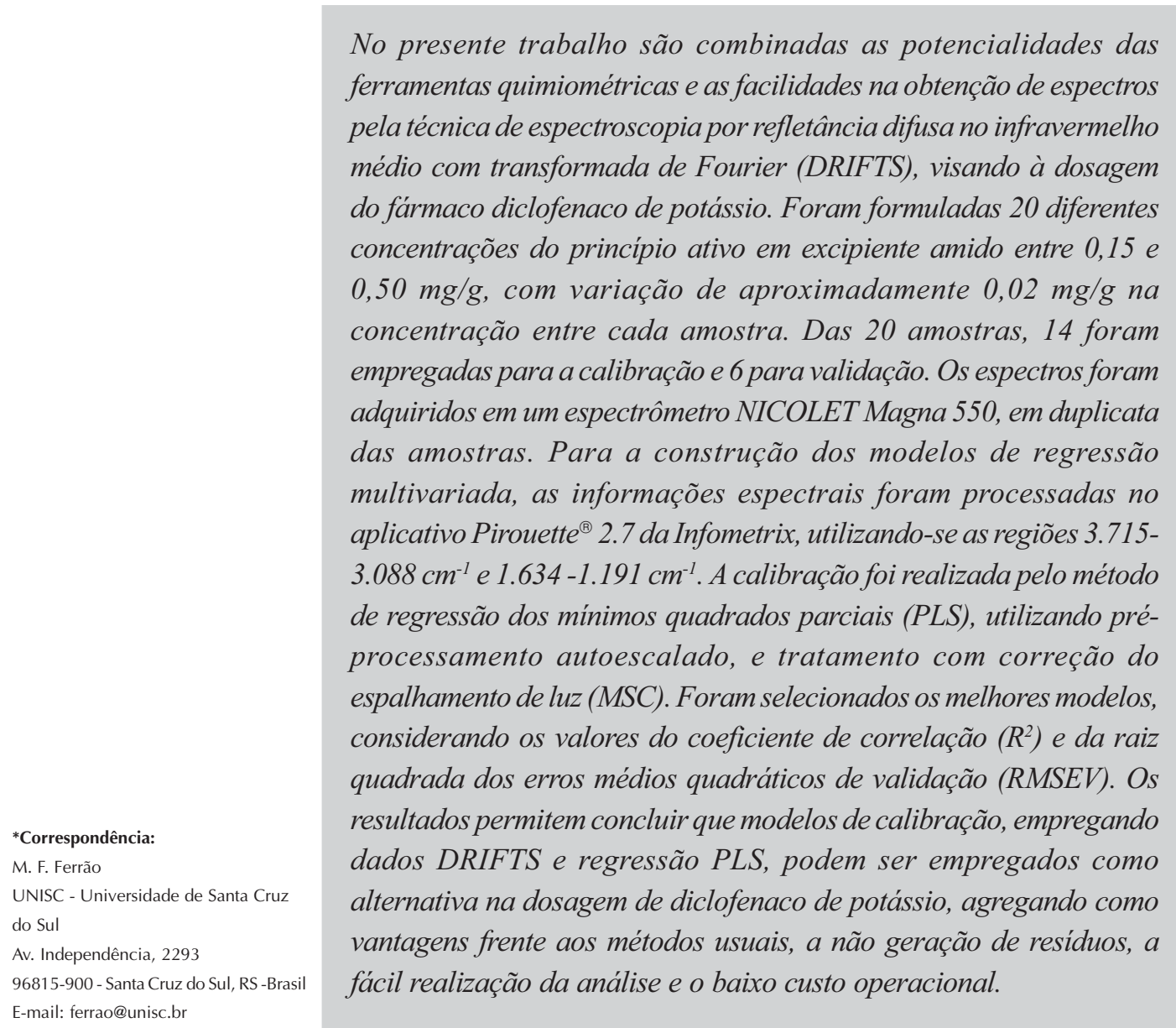

Unitermos

- Antiinflamatórios não-esteróides

- Diclofenaco

- Controle de qualidade

- Infravermelho

- Quimiometria

\section{INTRODUÇÃO}

A consciência da importância do controle de qualidade é histórica, pois, em 1352, João, o Bom, Rei da Fran- ça, legislou sobre a necessidade de serem controladas as preparações farmacêuticas (Prista, 1988). Com o desenvolvimento de novas técnicas analíticas o controle de qualidade evoluiu significativamente. 
Até recentemente, a espectroscopia na região do infravermelho era pouco utilizada em análises quantitativas em decorrência de algumas limitações inerentes à técnica. Porém, com a utilização da transformada de Fourier, aliada à evolução de técnicas quimiométricas, ao advento dos microprocessadores digitais e de técnicas computacionais avançadas, permitiu-se a análise de misturas complexas, como os medicamentos (De Souza, Ferrão, Parisotto, Furtado, 2004). Associada ao crescente desenvolvimento do método de refletância difusa, este tipo de análise garante a caracterização de fármacos com extrema rapidez. A identificação destas substâncias pode ser alcançada tanto quantitativamente como qualitativamente através da espectroscopia por refletância difusa no infravermelho com transformada de Fourier (Diffuse Reflectance Infrared Fourier Transform Spectroscopy - DRIFTS) (Yang, Irudayaraj, 2002), aliada a ferramentas quimiométricas de análise multivariada (Neumann et al., 2004; Parisotto et al., 2005).

No Brasil, uma característica evidente dos antiinflamatórios não-esteróides (AINEs) é o fato de serem medicamentos de venda livre e estarem em grande parte das associações medicamentosas irracionais disponíveis no mercado, além do fácil acesso devido ao grande número de apresentações disponíveis. O que acaba por caracterizar esta classe de medicamentos como a mais prescrita por médicos e dentistas, e conseqüentemente uma das mais consumidas. Fato este confirmado por diversos estudos sobre o uso de medicamentos no país, nos quais os antiinflamatórios não-esteróides figuram entre os primeiros colocados no ranking dos fármacos mais consumidos pela população (Ribeiro, Sevalho, Cesar, 2002).

Assim decidiu-se pela escolha deste fármaco como protagonista nesta pesquisa, visando à diversificação do método de controle de qualidade frente à elevação do número de fornecedores e ao aumento de produção deste medicamento, sendo necessário um maior monitoramento deste produto com o intuito de verificar a presença de medicamentos fora das especificações que poderiam comprometer as propriedades esperadas pelo consumidor.

\section{Antiinflamatórios não-esteróides: diclofenaco.}

Conhecidos pela humanidade há cerca de cem anos, os AINEs estão entre os agentes farmacológicos mais utilizados na prática médica (Kummer, Coelho, 2002). Até o presente, já existem mais de 50 AINEs diferentes no mercado, porém nenhum deles é ideal no controle ou na modificação dos sinais e sintomas da inflamação, particularmente àqueles que ocorrem nas do- enças articulares inflamatórias comuns. Praticamente todos os AINEs disponíveis no momento possuem efeitos indesejáveis significativos (Rang, Dale, Ritter, 2001).

O diclofenaco é utilizado em 120 países diferentes e por ventura o mais amplamente AINE utilizado no mundo. Existente há mais de 80 anos, se consolida no mercado mundial como a droga mais abundantemente vendida. Foi comercializado primeiramente no Japão em 1974, e introduzido nos Estados Unidos em 1989 (Foye, Lemke, Willians, 1995).

Estes antiinflamatórios são responsáveis por quase $25 \%$ das reações adversas a drogas notificadas oficialmente no Reino Unido. Também figuram nos relatórios de óbitos relacionados às drogas (Rang, Dale, Ritter, 2001).

\section{Identificação e Dosagem do Diclofenaco}

Existem diversas técnicas propostas na literatura para a identificação, caracterização e quantificação de diclofenaco de sódio e seus derivados (Palomo, Ballesteros, Frutos, 1999). Segundo a European Pharmacopoeia (2001) a identificação pode ser realizada através da espectroscopia no infravermelho ou por cromatografia em camada delgada comparando-se o tempo de retenção empregando-se amostras padrões. Já para análise quantitativa emprega-se a cromatografia líquida de alta eficiência com detector de UV (ultravioleta).

Recentes trabalhos apresentam a determinação de impurezas em formulações de diclofenaco através de polarografia (Kazemifard, Moore, Mohammadi, 2002). Já a determinação quantitativa tem sido proposta com detector de massas por cromatografia líquida de alta eficiência acoplado a espectrômetro de massa (Mayer et al., 2003) e por regressão multivariada empregando espectrofotometria UV (Sena et al., 2004). Apesar destas metodologias serem eficientes e recomendadas, em sua maior parte trata-se de métodos destrutivos, e apresentam longo tempo de análise.

\section{Espectroscopia por refletância difusa no infravermelho com transformada de Fourier}

A espectroscopia por refletância difusa é principalmente observada em amostras sólidas pulverizadas, sendo sua aplicação prática muito difundida na literatura, podendo ser utilizada em equipamentos que operam na região do infravermelho próximo (Blanco et al., 2001; Pasikatan et al., 2003; Pasquini, 2003, Steuer, Schulz, 2003; Sun, Xiang, An, 2004), associada a sigla NIRRS, Near Infrared Reflectance Spectroscopy, ou 
simplesmente NIRS. No final dos anos 70 e início dos 80 foi demonstrada a utilidade deste fenômeno quando acessórios de refletância difusa foram acoplados com espectrômetros interferométricos com transformada de Fourier (DRIFTS) (Fuller, Griffths, 1978).

A refletância difusa é observada quando uma luz incide em uma matriz descontínua, penetra na amostra (amostras do tipo pó, sólido, placa ou filme) e reflete trazendo informações espectrais. O caminho percorrido pela luz no interior da matriz pode ser considerado aleatório devido a múltiplas reflexões, algumas das quais após percorrer o interior de algumas partículas que constituem a amostra. Desta forma a luz refletida pode ser atenuada por absorção e o espectro resultante é similar ao obtido através da técnica no infravermelho por transmissão utilizando $\mathrm{KBr}$ (brometo de potássio). Uma importante diferença entre a transmissão e a refletância se dá devido o diferente caminho óptico percorrido pela luz. Enquanto que na transmissão o caminho óptico é constante para todo número de onda, na refletância por sua vez, o caminho pode ser variável. É de conhecimento geral que em regiões do espectro, onde a amostra absorve fracamente, a luz penetra mais profundamente na matriz, sendo que o contrário acontece onde há forte absorção. Portanto, ao se comparar o espectro obtido por transmissão (pastilha de $\mathrm{KBr}$ ) com o obtido por refletância, as intensidades relativas das bandas serão diferentes. Por exemplo, as bandas fracas no espectro por transmissão aparecerão mais fortes na refletância.

É importante salientar que num experimento de refletância difusa será também observada a reflexão especular, sendo mais importante àquela que ocorre na interface ar/superfície da matriz, mas também ocorrendo nas faces das partículas, mas cujo efeito pode ser minimizado pela redução do tamanho da partícula. A reflexão especular é de maior intensidade na região onde a amostra apresenta forte absorção e neste caso pode ocorrer severas distorções no espectro obtido (Ferrão, 2001). Deve-se ressaltar a diferença marcante entre infravermelho médio e próximo. No infravermelho próximo as distorções nos espectros são quase imperceptíveis, enquanto que no infravermelho médio as distorções são mais freqüentes. Uma maneira de contornar esse efeito não desejado é diluindo a amostra numa matriz não absorvente, como KBr (Olinger, Griffiths, 1993a, b; Pasikatan et al., 2001).

\section{Análise multivariada}

A análise multivariada é a rigor, qualquer abordagem analítica que considere o comportamento de duas ou mais variáveis simultaneamente, num vasto campo do conhecimento que envolve uma grande multiplicidade de conceitos estatísticos e matemáticos (Pereira, 1999).

Os métodos de análise multivariada aplicados na modelagem de informações químicas fazem parte da área da quimiometria, e são assim denominados pois no caso da espectroscopia permitem manipular dados de absorbância espectrais associadas a uma ou mais freqüências ao mesmo tempo. Estes métodos têm recentemente tornado possível modelar propriedades químicas e físicas de dados simples e complexos, a partir de dados espectroscópicos (Zamora et al., 1997).

\section{Regressão por mínimos quadrados parciais}

Este método foi desenvolvido nos anos 70 por Herman Wold, e um excelente guia prático foi escrito por Geladi e Kowalski (1986). Como usual em procedimentos analíticos primeiro desenvolve-se um modelo de calibração (Pimentel, Barros Neto, 1996). Uma matriz X com $\mathbf{m}$ colunas, correspondendo ao sinal espectral (p. e. absorbância) para cada número de onda, e n linhas, representando cada amostra (padrão). Uma segunda matriz $\mathbf{Y}$, é definida com $\mathbf{k}$ colunas, com as informações químicas (p. e. concentrações) e com $\mathbf{n}$ linhas, correspondendo a cada amostra. Utilizado técnicas de análise fatorial a matriz $\mathbf{X}$ é decomposta numa soma de matrizes menores (equação 1).

$$
\mathbf{X}=\mathbf{M}_{1}+\mathbf{M}_{2}+\ldots+\mathbf{M}_{\mathrm{a}}+\mathbf{E}(\text { Eq. 1) }
$$

Sendo $\mathbf{M}_{\mathrm{i}}$ os componentes principais ou variáveis latentes, com dimensão $1,2 \ldots a$, onde $a$ corresponde ao número de fatores (componentes principais ou variáveis latentes) selecionado para truncar a igualdade. E corresponde a matriz de resíduos, relacionada ao número de fatores.

A matriz Y é decomposta de maneira análoga. Finalmente, determina-se a correlação entre as variáveis latentes usando os componentes das matrizes menores.

No modelo PLS as decomposições das matrizes $\mathbf{X}$ e Y são feitas simultaneamente por meio interativo e se acredita que a informação contida em $\mathbf{Y}$ é transferida para $\mathbf{X}$.

Após a calibração é possível prever as propriedades de novas amostras a partir de medidas espectrais. Os sinais espectrais são definidos numa nova matriz $\mathbf{X}_{\mathrm{p}}$, que contém $\mathbf{m}$ colunas mas $\mathbf{t}$ linhas, correspondendo as $\mathbf{t}$ novas amostras. Usando a correlação construída na fase de calibração as propriedades desconhecidas são coletadas numa nova matriz $\mathbf{Y}_{\mathbf{p}}$. 


\section{MATERIAL E MÉTODOS}

\section{Amostragem}

Segundo a literatura, o diclofenaco de potássio (Figura 1), deve possuir não menos de $99 \%$ e não mais de $101,0 \%$ de potássio [sal potássico do ácido o-(2,6dicloroanilina)fenil acético] acetato (USP, 2000). Apresenta fórmula molecular $\mathrm{C}_{14} \mathrm{H}_{10} \mathrm{Cl}_{2} \mathrm{KNO}_{2}$, e peso molecular de 334,24g.mol ${ }^{-1}$ (European Pharmacopoéia, 2001).

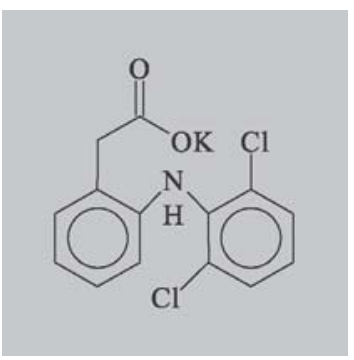

FIGURA 1 - Fórmula estrutural do diclofenaco de potássio (The Merck Index, 2001).

O princípio ativo diclofenaco de potássio (DK), foi manipulado juntamente com o excipiente amido, de forma a se obter um conjunto de amostras com diferentes concentrações, sendo pesadas quantidades calculadas de princípio ativo, e de amido separadamente, que posteriormente foram misturados até a obtenção de uma mistura homogênea. A variação da concentração deste conjunto foi de $0,15 \mathrm{mg} / \mathrm{g}$ acima e abaixo das concentrações usualmente comercializadas. Conferindo-se a variação de $0,02 \mathrm{mg} / \mathrm{g}$ para cada amostra, produzindo um grupo de 20 amostras. Cada amostra foi armazenada em frascos de vidro "snap-cap", que foram simultaneamente submetidos à homogeneização mecânica, por aproximadamente 3 horas.

\section{Aquisição dos espectros}

Os espectros das amostras foram obtidos em duplicata num espectrômetro Nicolet Magna 550 acoplando-se um acessório de refletância difusa EasiDiff ${ }^{\mathrm{TM}}$ da Pike Technologies, com resolução de $4 \mathrm{~cm}^{-1}$ e 32 varreduras, totalizando 40 espectros. Antes de cada amostra, foi obtido o espectro de referência (background) utilizando-se uma peça maciça de alumínio, cuja superfície foi previamente lixada, tornando-a fosca. O conjunto de espectros das amostras pode ser observado na Figura 2.

Previamente, foi selecionado o conjunto de regiões de maior interesse empregando o método de subtração de espectros, que consiste em subtrair os espectros de amos-

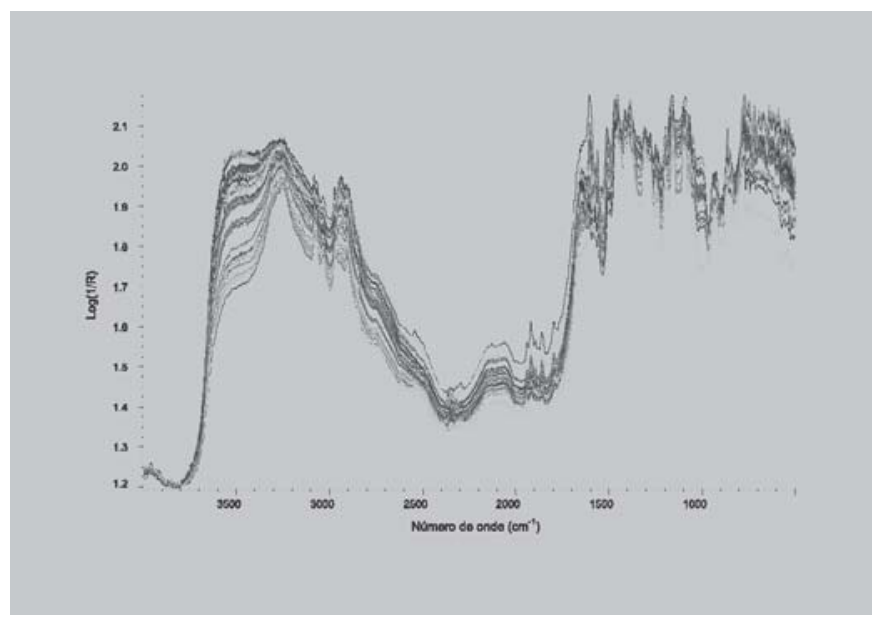

FIGURA 2 - Conjunto de espectros DRIFTS das amostras de diclofenaco de potássio.

tras com concentrações extremas do fármaco analisado, e a partir do perfil resultante, subdividir os espectros em sub-regiões que serão posteriormente empregadas na modelagem (Tabela I). Na Figura 3 são apresentados os sinais resultantes da subtração dos espectros das amostras de DK, contendo concentrações máxima e mínima.

TABELA I - Regiões selecionadas através do método de subtração de espectros para o princípio ativo DK

\begin{tabular}{ll}
\hline REGIÕES & SUB-REGIÃO \\
& DO DRIFTS $\left(\mathrm{cm}^{-1}\right)$ \\
\hline 1 & $3.715-3.247$ \\
2 & $3.245-3.088$ \\
3 & $1.634-1.430$ \\
4 & $1.428-1.271$ \\
5 & $1.256-1.191$ \\
\hline
\end{tabular}

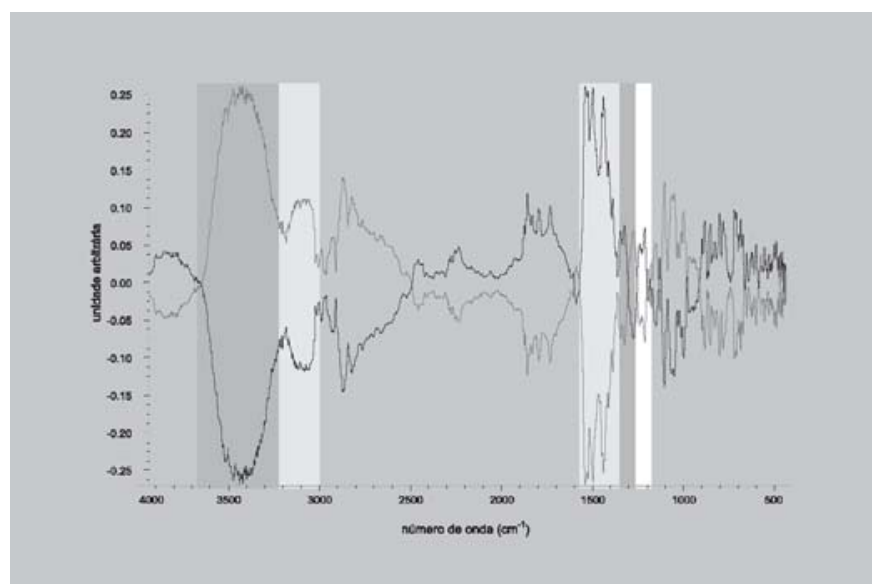

FIGURA 3 - Conjunto de perfis da subtração entre os espectros das amostras com alta e baixa concentrações de DK. 


\section{Análise Multivariada}

Para que um modelo seja eficiente, é necessário que ele descreva da melhor forma possível a situação real, levando-se em conta o maior número possível de variações. Quanto maior o número de fatores no modelo, menor será o desvio da reta de calibração. Porém, o aumento de fatores também aumenta o ruído e os erros de modelagem (Ferrão, Davanzo, 2005).

Geralmente o número apropriado de fatores, variáveis latentes (VLs), na calibração PLS pode ser determinada pelo PRESS (Predictive residual error sum of squares), que é a soma dos desvios quadrados para os valores estimados em relação aos valores atuais.

Outra medida que pode ser empregada para avaliar a habilidade de previsão de um modelo, o RMSEC (Root Mean Square Error of Calibration), ou simplesmente SEC (Standard Error of Calibration), que apresenta informações semelhantes aos do PRESS, sendo expressa conforme equação 02 , onde $n$ representa o número de amostras utilizadas.

$$
R M S E C=\sqrt{\frac{\sum_{i=l}^{n}\left(y_{i}-\hat{y}_{i}\right)^{2}}{n}} \text { (Eq. 2) }
$$

$\mathrm{Na}$ avaliação do conjunto de amostras de validação podemos ajustar a expressão empregada para determinar o SEC, sendo denominada de RMSEV (Root Mean Square Error of Validation), ou simplesmente SEV (Standard Error of Validation). Este critério é bastante útil quando se deseja avaliar se o modelo tende a superdimensionar o número de VLs a serem empregadas. Os valores de RMSEV, são calculados com os $y_{i}$ de referência e os valores estimados com o conjunto de validação, conforme equação 3 .

$$
R M S E V=\sqrt{\frac{\sum_{i=l}^{n}\left(y_{i}-\dot{y}_{i}\right)^{2}}{n}}
$$

Para a construção dos modelos de regressão, a matriz de dados espectrais foi autoescalada, sendo também aplicado o MSC. Utilizou-se o algoritmo PLS através do aplicativo Pirouette 2.7 da Infometrix para a modelagem dos dados espectrais, considerando como referência as concentrações previamente planejadas.

A determinação do número apropriado de fatores a serem utilizados, foi realizada pela análise do RMSEV, onde o modelo mais apropriado é alcançado quando se permite a utilização de um número mínimo destes fatores, com menor valor de RMSEV associado.

\section{RESULTADOS E DISCUSSÃO}

Diversos modelos de regressão foram construídos empregando-se o PLS, sendo variado o número de variáveis latentes (fatores) de 1 a 10, para a verificação das melhores regiões para realizar a calibração.

Foram calculados os valores de RMSEV para os modelos que empregaram cada uma das 5 regiões selecionadas, correlacionando o número de VLs, com a finalidade de acompanhar o desempenho destes modelos. A variação de RMSEV x n ${ }^{\circ}$ VLs, para todas as regiões espectrais estudadas, pode ser observada na Figura 4. $\mathrm{Na}$ Figura 5, observa-se gráfico das melhores regiões resultantes, 1,2 e 3 , bem como suas associações.

Pode-se observar que a utilização de poucos fatores, ressalta o modelo que usa a Região 1, empregando 1 fator, e também, o modelo que utiliza a combinação das Regiões 2 e 3, empregando 3 fatores. Nestes dois casos são observados baixos valores de erro para o conjunto de validação (RMSEV). Para estes modelos, também são apresentadas curvas de correlação entre os valores médios mensurados para a análise, e os valores médios obtidos via DRIFTS/PLS, bem como os valores de $\mathrm{R}^{2}$ e RMSEV.

As Figuras 6, e 7, apresentam curvas de correlação para a sub-região 1, e sub-regiões em associação 2 e 3, respectivamente.

Analisando-se os gráficos resultantes que apresentam as curvas de correlação das sub-regiões selecionadas, observa-se que os dois modelos desempenham perfis semelhantes, apesar de serem construídos com diferentes variáveis latentes.

Ressalta-se que a ordem de grandeza do $\mathrm{R}^{2}$ e do RMSEV, apresentam valores semelhantes nos dois modelos apresentados nas Figuras 6 e 7, mas discriminam o

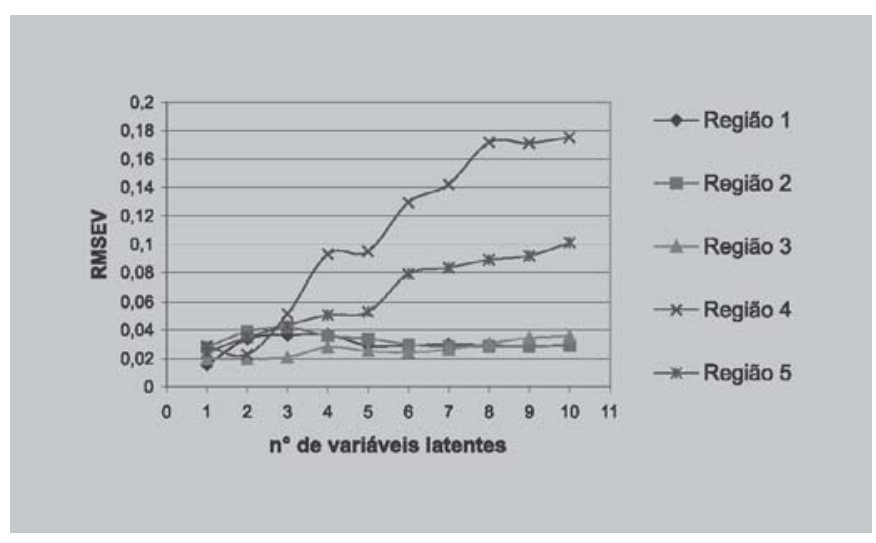

FIGURA 4 - Gráfico do RMSEV x nº de variáveis latentes, das regiões resultantes do método de subtração de espectros do fármaco DK. 


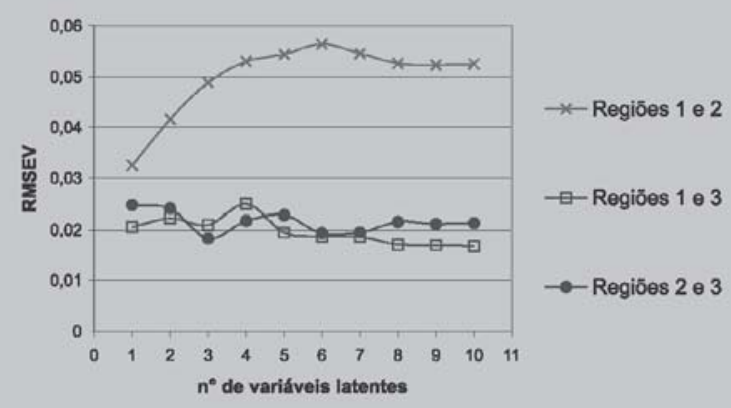

FIGURA 5 - Gráfico do RMSEV x n no de variáveis latentes, das associações das regiões 1,2 e 3 , selecionadas pelo método de subtração de espectros do fármaco DK.

modelo que emprega a região 1 , visto pequena variação diminuindo o RMSEV e aumentando o coeficiente de correlação, caracterizando-o como mais robusto, indicando com maior segurança a previsão das concentrações planejadas.

Para complementar este estudo, foram ainda calculadas, empregando o PLS Toolbox em ambiente MATLAB, a sensibilidade (SEN) e a seletividade (SEL) com base no Sinal Analítico Líquido (NAS) (Braga, Poppi, 2005), sendo os resultados apresentados na Tabela II.

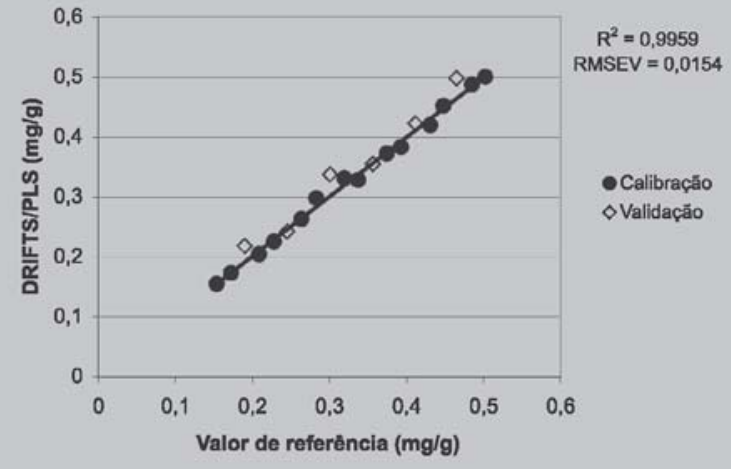

FIGURA 6 - Curva de correlação para o modelo que emprega os valores médios da sub-região 1, e seleção de uma variável latente.

Os valores de SEN não apresentaram diferença significativa o que já era esperado, uma vez que foram empregadas as mesmas condições experimentais (mesmo equipamento e acessório), para a aquisição da informação

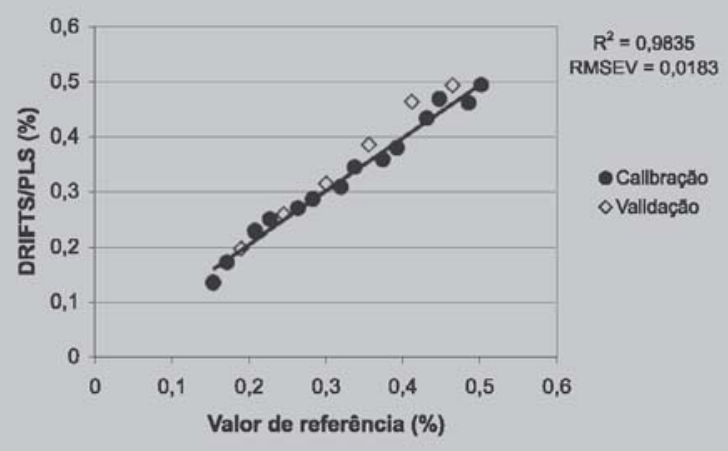

FIGURA 7 - Curva de correlação para o modelo que emprega os valores médios das sub-regiões associadas $2 \mathrm{e}$ 3 , e seleção de três variáveis latentes.

analítica. Já os valores de SEL reforçam o melhor desempenho dos modelos construídos com a região 1 e com a combinação das regiões 2 e 3 , uma vez que apresentaram maior seletividade tanto para os espectros de calibração quanto de validação.

TABELA II - Valores de sensibilidade e seletividade para os modelos de regressão multivariada empregando PLS.

\begin{tabular}{lcccc}
\hline Região(ões) & VL & SEN $^{\text {a }}$ & \multicolumn{2}{c}{ SEL } \\
& & & calibração & validação \\
\hline 1 & 1 & $5,2 \times 10^{-4}$ & 0,789 & 0,775 \\
2 & 1 & $5,1 \times 10^{-4}$ & 0,715 & 0,685 \\
3 & 1 & $5,0 \times 10^{-4}$ & 0,722 & 0,701 \\
4 & 2 & $5,3 \times 10^{-4}$ & 0,656 & 0,619 \\
5 & 1 & $5,2 \times 10^{-4}$ & 0,442 & 0,398 \\
1 e 2 & 1 & $4,8 \times 10^{-4}$ & 0,652 & 0,418 \\
1 e 3 & 1 & $4,9 \times 10^{-4}$ & 0,717 & 0,689 \\
2 e 3 & 3 & $4,9 \times 10^{-4}$ & 0,782 & 0,771 \\
\hline
\end{tabular}

$\mathrm{VL}=$ variáveis latentes; ${ }^{\mathrm{a}}$ valor expresso como a razão entre o $\log (1 / \mathrm{R})$ e $\mathrm{a} \%(\mathrm{~m} / \mathrm{m})$.

\section{CONCLUSÕES}

Verificou-se que a técnica DRIFTS associada do método de calibração PLS, se apresentou como excelente alternativa para a quantificação de diclofenaco de potássio, ratificando as potencialidades das técnicas de refletância que empregam regiões no infravermelho médio para a construção de modelos de previsão.

De uma forma geral este trabalho contribuiu para destacar as vantagens das técnicas espectroscópicas de refletância associadas às ferramentas quimiométricas, 
sobre os métodos oficiais de referência, como o baixo tempo de análise, a não destruição da amostra, o fácil manuseio dos instrumentos, a maior robustez do equipamento empregado visto a amplitude de amostras que comporta, e a não agressão ao meio ambiente por não gerar resíduos, bem como o baixo custo operacional frente a sua utilização diária em análises de rotina.

Todas estas vantagens agregam forte comprometimento na sustentação desta alternativa qualitativa e quantitativa, no monitoramento do processo produtivo de empresas do ramo farmacêutico que fabriquem ou manipulem fármacos em grande escala, sendo esta metodologia fácil de ser implantada em ambiente industrial visando sistemas de qualidade.

\section{AGRADECIMENTOS}

Os autores agradecem as bolsas de iniciação científica concedidas pela Fundação de Amparo a Pesquisa do Estado do Rio Grande do Sul e pelo apoio do Curso de Química e da Central Analítica da UNISC.

\section{ABSTRACT \\ Infrared spectroscopy applied to the drugs quality control. Part I: Multivariate regression for the quantification of potassium diclofenac}

In this work the alternative method for quantification of potassium diclofenac was studied, using diffuse reflectance infrared Fourier transform spectrocopy (DRIFTS), together with partial least squares regression (PLS). Two spectra of 20 samples with different concentrations $(0.15-0.50 \mathrm{mg} / \mathrm{g})$ of potassium diclofenac in starch had been colleted in Nicolet Magna 550 spectrophotometer, with resolution of the $4 \mathrm{~cm}^{-1}$ and 32 scans. For multivariate regression 14 samples had been used for calibration set and 6 samples had been used for validation set. For the PLS modeling, the spectral information (3715-3088 $\mathrm{cm}^{-1}$ and 1634-1191 $\left.\mathrm{cm}^{-1}\right)$ after multiplicative scatter correction (MSC) and autoscaling preprocess, had been processed in Pirouette ${ }^{\circledR}$ 2.7. The best models with better coefficient regression $\left(R^{2}\right)$, these were improved using root mean square error of validation (RMSEV). The new technique propose using DRIFTS/PLS shows an excellent choices for the quality control of the productive process of factories and drugstores that produce or handle these materials in large scale, presenting low time of analysis, no sample destruction and no wastes production.

UNITERMS: Non-steroidal anti-inflammatory drugs (NSAIDs). DRIFTS. Diclofenac. Drugs quality control. Chemometrics.

\section{REFERÊNCIAS}

BLANCO, M.; COELLO, J.; ITURRIAGA, H.; MASPOCH, S.; POU, N. Influence of the procedure used to prepare the calibration sample set on the performance of near infrared spectroscopy in quantitative pharmaceutical analyses. Analyst, London, v. 126, n. 7, p.1129-1134, 2001.

BRAGA, J.W.; POPPI, R.J. Validação de modelos de calibração multivariada: Uma aplicação na determinação de pureza polimórfica de carbamazepina por espectroscopia no infrevermelho próximo. Quím. Nova, São Paulo, v.27, p.1004-1011, 2004.

DE SOUZA, J.S.; FERRÃO, M.F.; PARISOTTO, G.; FURTADO, J.C. Análise de medicamentos antiinflamatórios não-esteróides manipulados empregando espectroscopia no infravermelho (DRIFTS). Rev. Cienc. Farm., Araraquara, v.25, n.2, p.91-98, 2004.

EUROPEAN PHARMACOPOÉIA. $4^{\text {th }}$ ed. Strasbourg: Council of Europe, 2001. p.1030-1031.

FERRÃO, M.F. Técnicas de reflexão no infravermelho aplicadas na análise de alimentos. Tecno-lóg., Santa Cruz do Sul, v.5, n.1, p.63-85, 2001.

FERRÃO, M.F.; DAVANZO; C.U. Horizontal attenuated total reflection applied to simultaneous determination of ash and protein contents in commercial wheat flour. Anal. Chim. Acta, v.440, n.2, p.411-415, 2005.

FOYE, W.O.; LEMKE, T.L.; WILLIANS, D.A. Principles of medicinal chemistry. 4. ed. Baltimore: Williams \& Wilkins, 1995.995 p.

FULLER, M.P.E.; GRIFFITHS, P.R. Diffuse reflectance measurements by infrared fourier transform spectrometry. Anal. Chem., Washington, v.50, p.1906-1910, 1978.

GELADI, P.E.; KOWALSKI, B.R. Partial least-squares regression: a tutorial. Anal. Chim. Acta, Amsterdam, v.185, p.1-17, 1986.

INFOMETRIX, Inc., Pirouette 2.70, Woodinville, WA, 1999.

KAZEMIFARD, A.G.; MOORE, D.E.; MOHAMMADI, A. Polarographic determination of benzaldehyde in benzyl alcohol and sodium diclofenac injection formulations. $J$. Pharm. Biomed. Anal., Amsterdam, v.30, n.2, p.257-262, 2002. 
KUMMER, C.L.; COELHO, T.C.R.B. Antiinflamatórios não esteróides inibidores da ciclooxigenase-2 (COX-2): Aspectos atuais. Rev. Bras. Anestesiol., Rio De Janeiro, v. 52, n. 4, p. 498-512, 2002.

MAYER, B.X.; NAMIRANIAN, K.; DEHGHANYAR, P.; STROH, R.; MASCHER, H.; MULLER, M. Comparison of UV and tandem mass spectrometric detection for the high-performance liquid chromatographic determination of diclofenac in microdialysis samples. J. Pharm. Biomed. Anal., Amsterdam, v.33, n.4, p.745-754, 2003.

NEUMANN, L.G.; FERRÃO, M.F.; PARISOTTO, G.; DE SOUZA, J.S.; KONZEN, P.H.A.; BRUXEL JUNIOR, V.; FURTADO, J.C. Algoritmo genético na otimização de HCA de espectros por reflexão difusa no infravermelho de medicamentos antiinflamatórios. Tecno-Lóg., Santa Cruz do Sul, v. 8, n.2, p.9-28, 2004.

OLINGER, J.M.E.; GRIFFITHS, P.R. Effects of sample dilution and particle size/morphology on diffuse reflection spectra of carbohydrate systems in the nearand mid-infrared. Part I: Single analytes. Appl. Spectrosc., Baltimore, v.47, p.687-694, 1993a.

OLINGER, J.M.E.; GRIFFITHS, P.R. Effects of sample dilution and particle size/morphology on diffuse reflection spectra of carbohydrate systems in the nearand mid-infrared. Part II: Durum wheat. Appl. Spectrosc., Baltimore, v. 47, p.695-701, 1993 b.

PALOMO, M.E.; BALLESTEROS, M.P.; FRUTOS P. Analysis of diclofenac sodium and derivates. J. Pharm. Biomed. Anal., Amsterdam, v.21, n.1, p.83-94, 1999.

PARISOTTO, G.; DE SOUZA, J.S.; FERRÃO, M.F.; FURTADO, J.C.; MOLZ, R.F. Análise exploratória aplicada no estudo de medicamentos contendo piroxicam. Rev. Bras. Ciênc. Farm., São Paulo, v. 41, n. 4, p. 499-505, 2005.

PASIKATAN, M.C.; STEELE, J.L.; SPILLMAN, C.K.; HAQUE E. Near infrared reflectance spectroscopy for online particle size analysis of powders and ground materials. J. Near Infrared Spectrosc., Sussex, v. 9, n.3, p. 153-164, 2001.

PASIKATAN, M.C.; HAQUE, E.; SPILLMAN, C.K.; STEELE, J.L.; MILLIKEN, G.A. Granulation sensing of first-break ground wheat using a near-infrared reflectance spectrometer: studies with soft red winter wheats. J. Sci. Food Agric., London, v.83, p.151-157, 2003.
PASQUINI, C. Near infrared spectroscopy: fundamentals practical aspects and analytical applications. J. Braz. Chem. Soc., São Paulo, v.14, n.2, p.138-219, 2003.

PEREIRA, J.C. Análise de dados qualitativos: estratégias metodológicas para as ciências da saúde, humanas e sociais. 2 ed. São Paulo: Editora da Universidade de São Paulo, 1999. 156 p.

PIMENTEL, M.F.; BARROS NETO, B. Calibração: Uma revisão para químicos analíticos. Quím. Nova, São Paulo, v.19, p.268-277, 1996.

PRISTA, L.N. Segurança e eficácia medicamentosa e controlo de qualidade. Rev. Port. Farm., Lisboa, v. 3, n. 38 , p. $1-8,1988$.

RANG, H.P.; DALE, M.M.; RITTER, J.M. Farmacologia. 4. ed. Rio de Janeiro: Guanabara Koogan, 2001. 703 p.

RIBEIRO, A.Q.; SEVALHO, G.; CESAR, C.C. Reações adversas gastrintestinais aos antiinflamatórios nãoesteróides - Uma abordagem em farmacoepidemiologia. Rev. Cienc. Farm, Araraquara, v. 23, n. 2, p. 175-198, 2002.

SENA, M.M.; CHAUDHRY, Z.F.; COLLINS, C.H.; POPPI, J.R. Direct determination of diclofenac in pharmaceutical formulations containing $B$ vitamins by UV spectrophotometry and partial least squares regression. $J$. Pharm. Biomed. Anal., Amsterdam, v.36, n.4, p.743-749, 2004.

STEUER, B.; SCHULZ, H. Near-infrared analysis of fennel (Foeniculum vulgare Miller) on different spectrometers - Basic considerations for a reliable network. Phytochem. Anal., Sussex, v.14, p.285-289, 2003.

SUN, M.L.; XIANG, B.R.; AN, D.K. A near-infrared diffuse reflectance analysis method for the noninvasive quantitative analysis of ambroxol hydrochloride tablets. Yао Хие Хие Bao, Peking, v.39, n.1, p.60-63, 2004.

THE MERCK INDEX: an encyclopedia of chemicals, drugs, and biologicals. Thirteenth edition. New Jersey: Merck, 2001, p.542.

USP 25 THE UNITED STATES PHARMACOPÉIA. 2002. NF 20 THE NATIONAL FORMULARY. Washington, D.C: United States Pharmacopeial Convention, INC., 2000. p.553-554. 
YANG, H.; IRUDAYARAJ, J. Rapid determination of vitamin $C$ by NIR, MIR and FT-Raman techniques. $J$. Pharm. Pharmacol., London, v.54, n.9, p.1247-1255, 2002.
ZAMORA, P.P.; PONCE, L.C.; NAGATA, N.; POPPI, R.J. Alternativas quimiométricas para a resolução de problemas analíticos clássicos. Determinação espectrofotométrica de misturas de zircônio e háfnio. Quím. Nova, São Paulo, v. 20, n. 5, p. 469-474, 1997.

Recebido para publicação em 28 de julho de 2005. Aceito para publicação em 06 de julho de 2006. 